\title{
A New Numerical Method for Calculating Extrema of Received Power for Polarimetric SAR
}

\author{
Yonghong Zhang, Jixian Zhang, Zhong Lu, Senior Member, IEEE, and Wenyu Gong
}

\begin{abstract}
A numerical method called cross-step iteration is proposed to calculate the maximal/minimal received power for polarized imagery based on a target's Kennaugh matrix. This method is much more efficient than the systematic method, which searches for the extrema of received power by varying the polarization ellipse angles of receiving and transmitting polarizations. It is also more advantageous than the Schuler method, which has been adopted by the PolSARPro package, because the cross-step iteration method requires less computation time and can derive both the maximal and minimal received powers, whereas the Schuler method is designed to work out only the maximal received power. The analytical model of received-power optimization indicates that the first eigenvalue of the Kennaugh matrix is the supremum of the maximal received power. The difference between these two parameters reflects the depolarization effect of the target's backscattering, which might be useful for target discrimination.
\end{abstract}

Index Terms-Polarimetry, scattering, scattering parameter measurements, synthetic aperture radar (SAR).

\section{INTRODUCTION}

$\mathbf{P}$ OLARIMETRIC synthetic aperture radar (SAR) data are receiving more attention following the recent launch of several new-generation SAR satellites (Advanced Land Observation System Phased-Array L-Band SAR, TerraSAR, and Radarsat-2) with polarimetric capabilities. It can be expected that the rich polarimetric SAR data resources will stimulate more interest in developing new algorithms and methodologies for information extraction from the polarized signal.

A polarimetric SAR measures the microwave reflectivity of a target using quad-polarizations $\mathrm{HH}, \mathrm{HV}, \mathrm{VH}$, and $\mathrm{VV}$ to form a scattering matrix [1]. Having measured this matrix, the target response to any combination of transmitted and received polarizations can be calculated through polarization synthesis [2]. There are specific transmitting/receiving polarizations that maximize and minimize the power backscattered from the target and received by the antenna. These specific polarizations are called optimal polarization states. The corresponding maxima and minima of the received power are basic parameters

Manuscript received November 19, 2008; revised March 30, 2009. First published July 31, 2009; current version published October 14, 2009. This work was supported by the National Key Basic Research and Development Program, China, under Project 2006CB701303.

Y. Zhang, J. Zhang, and W. Gong are with the Chinese Academy of Surveying and Mapping, Beijing 100039, China (e-mail: yhzhang@ casm.ac.cn; zhangjx@casm.ac.cn; gongwy@casm.ac.cn).

Z. Lu is with the U.S. Geological Survey, Earth Resources Observation and Science Center and Cascades Volcano Observatory, Vancouver, WA 98683 USA (e-mail: lu@usgs.gov).

Color versions of one or more of the figures in this paper are available online at http://ieeexplore.iee.org.

Digital Object Identifier 10.1109/LGRS.2009.2022958 for polarized SAR data and contain important information to discriminate different types of targets [3], [4].

The optimal polarization problem can easily be solved if the scattered wave is assumed to be completely polarized. Under this assumption, the maximal received power, which is identical to the maximal backscattered power, corresponds to the maximal eigenvalue of the Graves matrix [3] or the covariance matrix [5]. However, in practice, the scattered wave is usually partially rather than completely polarized because a natural target consisting of distributed scatterers has a depolarization effect. In this case, the maximization of the received power is equivalent to numerically solving a sixth-order polynomial equation [4], [6].

In this letter, we propose a new numerical method, which we call the cross-step iteration method, to calculate the maximum and the minimum of polarimetric received powers directly from the Kennaugh matrix. This method is much more computationally efficient compared with the systematic method, which consists of deriving the extrema from the polarization ellipse angles sampled at a small interval. We present the analytic formulation of the received-power optimization problem in Section II. The proposed cross-step iteration method is detailed in Section III. Section IV presents an example of calculating the maximal and minimal received powers with AIRSAR L-band polarimetric data. The proposed method is compared not only with the systematic method but also with a widely used computation method, which has been adopted by PolSARPro, a polarimetric SAR data processing and educational package developed by the European Space Agency [7]. The relationship between the maximal/minimal received power and the maximum eigenvalue of the polarimetry Kennaugh matrix, as well as their implications, is discussed in this section. Conclusions are drawn in Section V.

\section{AnAlytic Formulation}

For a target characterized by its $4 \times 4$ Kennaugh matrix $\mathbf{K}$, the backscattered power from the target can be defined as in [8], i.e.,

$$
P=\frac{1}{2} G_{r} \cdot \mathbf{K} G_{t}
$$

where $G_{r}$ and $G_{t}$ are the Stokes vectors of receiving and transmitting polarizations, respectively. Here, we have ignored a systematic constant depending on the antenna gain, transmitted wavelength, and permittivity and permeability of free space [3]. The Kennaugh matrix is related to the scattering matrix $\mathbf{S}$ as in [9], i.e.,

$$
\mathbf{K}=\mathbf{A}^{*}\left(\mathbf{S} \otimes \mathbf{S}^{*}\right) \mathbf{A}^{-1}
$$


where “ $\otimes$ " and superscript “*” symbolize the standard Kronecker matrix product and complex conjugate, respectively, and $\mathbf{A}$ is the Kronecker expansion matrix.

It should be noted that in the backscattering case, for most natural targets, reciprocity holds; therefore, the scattering matrix is symmetric. Thus, the Kennaugh matrix defined in (2) is real and symmetric.

When considering a completely polarized wave, the normalized Stokes vector has the form

$$
G=\left[\begin{array}{c}
1 \\
\cos (2 \varphi) \cos (2 \chi) \\
\sin (2 \varphi) \cos (2 \chi) \\
\sin (2 \chi)
\end{array}\right] .
$$

Here, $\varphi$ and $\chi$ denote the orientation and ellipticity angles of the polarization wave, respectively.

According to the Cauchy inequality, the backscattered power in (1) satisfies

$$
P \leq \frac{1}{2}\left\|G_{r}\right\|_{2}\left\|\mathbf{K} G_{t}\right\|_{2}=\frac{\sqrt{2}}{2}\left\|\mathbf{K} G_{t}\right\|_{2} .
$$

Here, \|\|$_{2}$ denotes the two-norm of vectors, e.g., the vector length in Euclidean space.

Since the spectral norm of matrices is compatible with the two-norm of vectors [10], the above inequality becomes

$$
P \leq \frac{\sqrt{2}}{2}\left\|\mathbf{K} G_{t}\right\|_{2} \leq \frac{\sqrt{2}}{2}\left\|G_{t}\right\|_{2}\|\mathbf{K}\|_{\lambda}=\|\mathbf{K}\|_{\lambda}
$$

where \|\|$_{\lambda}$ represents the spectral norm of matrices. As the Kennaugh matrix is real and symmetric, its spectral norm is equal to its maximal or first eigenvalue [10]. This means that the first eigenvalue of a target's Kennaugh matrix is the supremum of the optimal received power from the target. The equality can only be achieved when the scattered wave represented by $\mathbf{K} G_{t}$ is completely polarized. In that case, the maximum received power can be obtained if the Stokes vectors of both transmitting and receiving polarizations are set to be identical to the eigenvector of $\mathbf{K}$ for its first eigenvalue.

\section{Cross-Step Iteration Method}

The cross-step iteration method basically uses the Cauchy inequality to construct a transmitting/receiving polarization for a given receiving/transmitting polarization state to achieve a maximal (or minimal) received power.

Let us rewrite the Stokes vector of transmitting and receiving polarizations as

$$
G_{r}=\left[\begin{array}{llll}
1 & r_{1} & r_{2} & r_{3}
\end{array}\right]^{T} \quad G_{t}=\left[\begin{array}{llll}
1 & t_{1} & t_{2} & t_{3}
\end{array}\right]^{T}
$$

with

$$
r_{1}^{2}+r_{2}^{2}+r_{3}^{2}=1 \quad t_{1}^{2}+t_{2}^{2}+t_{3}^{2}=1
$$

where the superscript $T$ denotes transpose.

For a given transmitting polarization state $G_{t}$, if we record $A=\mathbf{K} G_{t}=\left[\begin{array}{llll}A_{0} & A_{1} & A_{2} & A_{3}\end{array}\right]^{T}$, then the received power is

$$
P=G_{r} \cdot A / 2=\left(A_{0}+r_{1} A_{1}+r_{2} A_{2}+r_{3} A_{3}\right) / 2 .
$$

Applying the Cauchy inequality, we can get

$$
P \leq\left(A_{0}+\sqrt{A_{1}^{2}+A_{2}^{2}+A_{3}^{2}}\right) / 2 .
$$

The equality can be achieved only if

$$
r_{i}=A_{i} / \sqrt{A_{1}^{2}+A_{2}^{2}+A_{3}^{2}}, \quad i=1,2,3 .
$$

At the same time, the received power has a lower bound

$$
P \geq\left(A_{0}-\sqrt{A_{1}^{2}+A_{2}^{2}+A_{3}^{2}}\right) / 2 .
$$

The equality can be achieved only if

$$
r_{i}=-A_{i} / \sqrt{A_{1}^{2}+A_{2}^{2}+A_{3}^{2}}, \quad i=1,2,3 .
$$

On the other hand, the received power can be rewritten as

$$
P=\frac{1}{2} G_{t} \cdot \mathbf{K} G_{r} .
$$

If we record $B=\mathbf{K} G_{r}=\left[\begin{array}{llll}B_{0} & B_{1} & B_{2} & B_{3}\end{array}\right]^{T}$, then (12) can be rewritten as

$$
P=G_{t} \cdot B / 2=\left(B_{0}+t_{1} B_{1}+t_{2} B_{2}+t_{3} B_{3}\right) / 2 .
$$

Once the receiving polarization state $G_{r}$ is fixed, similarly, the maximal received power can be achieved only if the transmitting polarization state satisfies

$$
t_{i}=B_{i} / \sqrt{B_{1}^{2}+B_{2}^{2}+B_{3}^{2}}, \quad i=1,2,3 .
$$

At the same time, the minimal received power can be achieved under the condition

$$
t_{i}=-B_{i} / \sqrt{B_{1}^{2}+B_{2}^{2}+B_{3}^{2}}, \quad i=1,2,3 .
$$

The cross-iteration method for the optimal received-power problem can be summarized in the following steps.

1) The method starts with an initial $\mathrm{H}$ or $\mathrm{V}$ polarization as the transmitting polarization state, e.g., $\mathrm{H}$ polarization with $G_{t}^{(0)}=\left[\begin{array}{llll}1 & t_{1}^{(0)} & t_{2}^{(0)} & t_{3}^{(0)}\end{array}\right]^{T}=\left[\begin{array}{llll}1 & 1 & 0 & 0\end{array}\right]^{T}$.

2) Knowing $G_{t}^{(n)}=\left[\begin{array}{llll}1 & t_{1}^{(n)} & t_{2}^{(n)} & t_{3}^{(n)}\end{array}\right]^{T}$, we consider the maximization problem $\max \left(G_{r} \cdot \mathbf{K} G_{t}^{(n)}\right)$. From the above discussion, we know that if the receiving polarization $G_{r}^{(n+1)}$ is set as (9), $\left(G_{r}^{(n+1)} \cdot \mathbf{K} G_{t}^{(n)}\right)$ can achieve the maximum.

3) When the receiving polarization is known, the optimal received-power problem becomes finding an appropriate transmitting polarization $G_{t}$ to maximize $\left(G_{r}^{(n+1)}\right.$. $\left.\mathbf{K} G_{t}\right)$. Again, the solution is to have the transmitting polarization $G_{t}^{(n+2)}$ defined as (14).

4) The cross-step iteration will stop if $\sum_{i=1}^{3} \mid t^{(n+2)}-$ $t^{(n)} \mid \leq \xi$ and $\sum_{i=1}^{3}\left|r^{(n+1)}-r^{(n-1)}\right| \leq \xi$ are both true. Here, $\xi$ is a predefined indefinite positive number, e.g., $\xi=1.0 \times 10^{-5}$. At that time, the received power comes to a local maximum. 


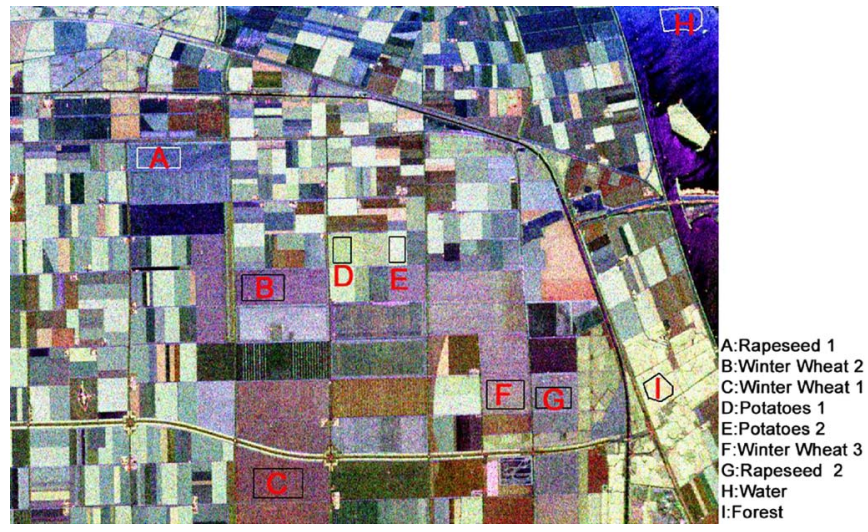

Fig. 1. AIRSAR L-band data over Flevoland region, The Netherlands. The false-color image is derived by assigning $\left|S_{\mathrm{hh}}-S_{\mathrm{vv}}\right|^{2} / 2,2\left|S_{\mathrm{hv}}\right|^{2}$, and $\left|S_{\mathrm{hh}}+S_{\mathrm{vv}}\right|^{2} / 2$ as RGB channels, respectively; here $S_{\mathrm{hh}}, S_{\mathrm{hv}}$, and $S_{\mathrm{vv}}$ are the elements of the scattering matrix $\mathbf{S}$, and || represents the module of a complex number. Nine AOIs belonging to five land-cover classes were drawn manually by referring a land-cover ground-truth map.

5) Repeat steps 1-4 with $\mathrm{V}$ polarization as the initial trans-

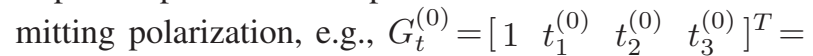
$\left[\begin{array}{llll}1 & -1 & 0 & 0\end{array}\right]^{T}$. Then, another local maximum will be obtained. The larger of these two local maxima is the final maximal (global maximal) received power, and the corresponding transmitting and receiving polarization states will be recorded.

Similarly, cross-step iteration steps can be formed to calculate the minimum received power.

\section{EXPERIMENT AND DISCUSSION}

To demonstrate the validity of the proposed new method, an experiment was conducted with AIRSAR L-band data. In the experiment, the optimal received power of different terrain-cover types was calculated with three methods: the proposed cross-step iteration method, the systematic method, and a widely used computation method. The systematic method calculates and compares the received powers for various transmitting and receiving polarizations using the polarization orientation $\varphi$ and the ellipticity angle $\chi$ sampled at intervals of $0.1^{\circ}$. The widely used method first calculates the eigenvalues of the Graves or polarization power scattering matrix, then forms the cross-polarization ratio associated with the eigenvalue, and, finally, starts gradient-descend-optimization iterations to calculate the maximal received power with an initial Stokes vector based on the polarization ratio. This method seems more computationally efficient than the aforementioned method with solving a sixth-order polynomial equation. It will be called the Schuler method hereafter since the algorithm was presented in detail by Schuler and Lee [11]. However, in truth, the principle of this method had been discussed earlier by Boerner et al. [1].

The AIRSAR data used in the experiment is a quadpolarized image over an agricultural region in Flevoland, The Netherlands, acquired in August 1989. The image has been multilook-processed and has a pixel size of about $12 \times 12 \mathrm{~m}$. Nine areas of interest (AOIs) belonging to five different landcover types were selected (see Fig. 1). For each AOI, the mean coherence matrix is estimated. Then, the maximal received power for each AOI is calculated using the cross-step iteration method, the systematic method, and the Schuler method, respectively. The result is shown in Table I. As a comparison, the first eigenvalue of the corresponding mean Kennaugh matrix of each AOI is listed at the last column of Table I. The first eigenvalue is larger than the maximal received power for every AOI. The cross-step iteration method gives nearly the same maximal received power as the Schuler method for all terraincover types. The two both derive a little larger optimal received power than the systematic method. The reason is that the polarization orientation and ellipticity angles corresponding to the maximal received power derived by the two methods may not be achieved by a $0.1^{\circ}$ sampling interval for polarization orientation and ellipticity angles in the systematic method.

Table I also lists the number of iterations and the computation time used by the three methods. Because the cross-step iteration method and the Schuler method needed only a small number of iterations, the computation time used for each AOI alone was too short to be measurable. Alternatively, the aggregate time consumed for the nine terrain-cover types is listed for these two methods. Obviously, the systematic method needed many more iterations and longer computation times, which are directly related to the specified sampling intervals of $\varphi$ and $\chi$ angles. Increasing the step length will decrease both the number of iterations and the computation time, but may not achieve the desired accuracy. Table $I$ indicates that a $0.1^{\circ}$ sampling interval is a good choice and brings out very accurate maximal received power. Compared with the Schuler method, the cross-step iteration method requires a similar number of iterations for every AOI but saves $34 \%$ of computation time to derive the maximal received power for the total nine AOIs. The main reason is because the Schuler method involves calculating the eigenvalues of the Graves matrix before starting iteration, whereas the cross-step iteration method does not need this extra process. More importantly, the Schuler method can only work out the maximal received power, whereas the cross-step iteration method can work out both the maximal and minimal received powers. This will be explained in detail in the next paragraph.

A polarimetric SAR transmits a completely polarized electromagnetic wave, which, being scattered off a distributed target, results in a partially polarized scattered wave [6]. A partially polarized wave can be considered as the superposition of a completely polarized wave and an unpolarized wave. The received power from the completely polarized component can be optimized by adjusting the receiving polarization, but the antenna will always receive half the available power from the unpolarized component, irrespective of the polarization of the receiving antenna [1]. Therefore, the maximum received power will be reached when the received power from the completely polarized part of the backscattered wave is maximized. For the completely polarized case, the maximum received power is achieved when the transmitting and receiving antennas are identical and equal to the eigenvector of the Graves matrix corresponding to the maximal eigenvalue [3]. Therefore, generally, to maximize the received power, the transmitting and receiving polarizations have to be the same. This implies a favorable prerequisite for the maximal polarization problem, which has been taken advantage of by both the Schuler method and the method with solving a sixth-order polynomial equation. 
TABLE I

Maximal Received Powers Calculated by Three Different Methods and the First Eigenvalue of the Corresponding Kennaugh Matrix for Nine Selected AOIs in Fig. 1

\begin{tabular}{|c|c|c|c|c|c|c|c|c|c|c|}
\hline \multirow[b]{2}{*}{ AOI } & \multicolumn{3}{|c|}{ Cross-step iteration } & \multicolumn{3}{|c|}{ Schuler method } & \multicolumn{3}{|c|}{ Systematic method } & \multirow[b]{2}{*}{$\lambda_{1}(\mathbf{K})$} \\
\hline & $P_{\max }$ & $\begin{array}{c}\text { Number of } \\
\text { iterations }\end{array}$ & $\begin{array}{c}\text { Computation } \\
\text { time }\end{array}$ & $P_{\max }$ & \begin{tabular}{|c|} 
Number of \\
iterations
\end{tabular} & $\begin{array}{c}\text { Computation } \\
\text { time }\end{array}$ & $P_{\max }$ & \begin{tabular}{|c|} 
Number of \\
iterations
\end{tabular} & $\begin{array}{c}\text { Computation } \\
\text { time }\end{array}$ & \\
\hline Rapeseed 1 & $7.300058 \mathrm{E}-03$ & 77 & \multirow[t]{9}{*}{$0.256 \mathrm{sec}$} & $7.300058 \mathrm{E}-03$ & 70 & \multirow[t]{9}{*}{$0.389 \mathrm{sec}}$. & $7.300056 \mathrm{E}-03$ & 1648910 & $0.703 \mathrm{sec}$ & 7.324984E-03 \\
\hline Rapeseed 2 & $7.104888 \mathrm{E}-03$ & 44 & & 7.104889E-03 & 50 & & $7.104887 \mathrm{E}-03$ & 1648910 & $0.687 \mathrm{sec}$. & $7.150418 \mathrm{E}-03$ \\
\hline \begin{tabular}{|l} 
Winter \\
wheat 1 \\
\end{tabular} & $3.588609 \mathrm{E}-03$ & 49 & & $3.588609 \mathrm{E}-03$ & 58 & & $3.588609 \mathrm{E}-03$ & 1648910 & $0.687 \mathrm{sec}$ & 3.603576E-03 \\
\hline $\begin{array}{l}\text { Winter } \\
\text { wheat } 2 \\
\end{array}$ & 7.321957E-03 & 31 & & $7.321956 \mathrm{E}-03$ & 40 & & $7.321953 \mathrm{E}-03$ & 1648910 & $0.688 \mathrm{sec}$ & 7.334181E-03 \\
\hline $\begin{array}{l}\text { Winter } \\
\text { wheat } 3 \\
\end{array}$ & $7.874088 \mathrm{E}-03$ & 24 & & 7.874088E-03 & 34 & & $7.874083 \mathrm{E}-03$ & 1648910 & $0.672 \mathrm{sec}$ & $7.947210 \mathrm{E}-03$ \\
\hline Potatoes 1 & $1.855846 \mathrm{E}-02$ & 75 & & $1.855847 \mathrm{E}-02$ & 67 & & $1.855846 \mathrm{E}-02$ & 1648910 & $0.687 \mathrm{sec}$. & 2.046507E-02 \\
\hline Potatoes 2 & 7.309211E-03 & 117 & & 7.309211E-03 & 120 & & $7.309209 \mathrm{E}-03$ & 1648910 & $0.688 \mathrm{sec}$. & $8.916170 \mathrm{E}-03$ \\
\hline Forest & $1.345572 \mathrm{E}-02$ & 22 & & $1.345572 \mathrm{E}-02$ & 31 & & $1.345572 \mathrm{E}-02$ & 1648910 & $0.672 \mathrm{sec}$ & $1.677559 \mathrm{E}-02$ \\
\hline Water & $1.691631 \mathrm{E}-02$ & 86 & & $1.691630 \mathrm{E}-02$ & 94 & & $1.691630 \mathrm{E}-02$ & 1648910 & $0.687 \mathrm{sec}$. & 1.691957E-02 \\
\hline
\end{tabular}

TABLE II

Transmitting And Receiving Polarizations Corresponding to Maximal ReCEIVED Powers Calculated By Three Different Methods. The Unit Is In Degrees; Subscript “T” Denotes Transmitting, Whereas “R” Denotes Receiving

\begin{tabular}{|l|c|c|c|c|c|}
\hline \hline \multirow{2}{*}{ AOI } & \multicolumn{2}{|c|}{ Cross-step iteration } & \multicolumn{2}{c|}{ Schuler method } & \multicolumn{2}{c|}{ Systematic method } \\
\cline { 2 - 6 } & $\left(\varphi_{t}, \chi_{t}\right)$ & $\left(\varphi_{r}, \chi_{r}\right)$ & $\left(\varphi_{t,}, \chi_{t}\right)$ & $\left(\varphi_{r,}, \chi_{r}\right)$ \\
\hline Rapeseed 1 & $(92.241348,-0.581741)$ & $(92.241257,-0.581747)$ & $(92.249717,-0.581236)$ & $(92.2,-0.6)$ & $(92.2,-0.6)$ \\
\hline Rapeseed 2 & $(89.325424,-0.476999)$ & $(89.325371,-0.477010)$ & $(89.322707,-0.477477)$ & $(89.3,-0.5)$ & $(89.3,-0.5)$ \\
\hline Winter wheat 1 & $(90.489914,0.885107)$ & $(90.489876,0.885098)$ & $(90.489037,0.884518)$ & $(90.5,0.9)$ & $(90.5,0.9)$ \\
\hline Winter wheat 2 & $(88.790779,-0.659205)$ & $(88.790855,-0.659189)$ & $(88.793607,-0.658977)$ & $(88.8,-0.7)$ & $(88.8,-0.6)$ \\
\hline Winter wheat 3 & $(96.136887,4.959248)$ & $(96.136925,4.959254)$ & $(96.134892,4.958828)$ & $(96.1,5.0)$ & $(96.1,4.9)$ \\
\hline Potatoes 1 & $(172.685806,-0.106074)$ & $(172.685715,-0.106075)$ & $(172.678438,-0.106200)$ & $(172.7,-0.1)$ & $(172.7,-0.1)$ \\
\hline Potatoes 2 & $(82.036652,-0.668142)$ & $(82.036758,-0.668138)$ & $(82.052463,-0.667608)$ & $(82.0,-0.7)$ & $(82.0,-0.7)$ \\
\hline Forest & $(86.193748,-1.377421)$ & $(86.193794,-1.377432)$ & $(86.196584,-1.378136)$ & $(86.2,-1.4)$ & $(86.2,-1.4)$ \\
\hline Water & $(78.840431,-0.476921)$ & $(78.840530,-0.476915)$ & $(78.853721,-0.476496)$ & $(78.8,-0.5)$ & $(78.8,-0.5)$ \\
\hline \hline
\end{tabular}

TABLE III

Minimal Received Powers With Corresponding TransmitTing And Receiving Polarizations DeRived With the Cross-Step Iteration Method, And Two Derived Parameters

\begin{tabular}{|l|l|l|l|l|c|c|}
\hline \hline \multicolumn{1}{|c|}{ AOI } & \multicolumn{1}{c|}{$P_{\min }$} & \multicolumn{1}{c|}{$\left(\varphi_{t,}, \chi_{t}\right)$} & \multicolumn{1}{c|}{$\left(\varphi_{r,}, \chi_{r}\right)$} & $D_{p}$ & $\left(P_{\min }+P_{\max }\right) / \lambda_{1}(\mathbf{K})$ \\
\hline Rapeseed 1 & $2.570737 \mathrm{E}-04$ & $(95.104309,0.667657)$ & $(4.505462,1.174420)$ & 0.00340 & 0.93197 & 1.031692 \\
Rapeseed 2 & $4.207024 \mathrm{E}-04$ & $(86.876907,-1.868767)$ & $(177.732788,0.315554)$ & 0.00637 & 0.88819 & 1.052469 \\
Winter wheat 1 & $1.601840 \mathrm{E}-04$ & $(89.122551,0.164337)$ & $(179.856430,-0.716160)$ & 0.00415 & 0.91454 & 1.040298 \\
Winter wheat 2 & $2.240196 \mathrm{E}-04$ & $(88.978996,-0.840181)$ & $(178.851486,0.544822)$ & 0.00167 & 0.94063 & 1.028878 \\
Winter wheat 3 & $4.890904 \mathrm{E}-04$ & $(89.850471,2.809876)$ & $(3.005262,-4.556826)$ & 0.00920 & 0.88304 & 1.052341 \\
Potatoes 1 & $3.492795 \mathrm{E}-03$ & $(79.119560,0.453670)$ & $(167.648224,0.954186)$ & 0.09316 & 0.68321 & 1.077507 \\
Potatoes 2 & $2.020587 \mathrm{E}-03$ & $(90.033104,-1.524286)$ & $(178.771286,0.314048)$ & 0.18023 & 0.56685 & 1.046391 \\
Forest & $3.484342 \mathrm{E}-03$ & $(90.242447,-0.133520)$ & $(178.171906,1.506139)$ & 0.19790 & 0.58863 & 1.009804 \\
Water & $5.763529 \mathrm{E}-05$ & $(106.656197,3.669305)$ & $(12.446294,3.725804)$ & 0.00019 & 0.99321 \\
\hline \hline
\end{tabular}

Table II lists the transmitting and receiving polarization angles corresponding to the maximal received power calculated with the three methods. Although the condition that the transmitting polarization should be equal to the receiving polarization is not a prerequisite for cross-step iteration and systematic methods, this condition is actually being precisely obeyed by the two methods, as shown in Table II. However, such a specific relationship between the transmitting and receiving polarizations does not exist when the minimal received power is considered. Therefore, it is impossible to give an analytic closedform solution for the minimum received power problem. This is why the Schuler method can only work out the maximal received power. For the same reason, Touzi et al. [4] derived the minimal received power based on some assumptions about the relationship between minimal and maximal received powers, rather than directly calculated this value.

The minimal received power can also be efficiently calculated with the cross-step iteration method. Table III shows the minimal received power and the corresponding transmitting and receiving polarizations calculated with this method. We cannot see a clear relationship between the transmitting and receiving polarizations over which the minimal received power is achieved. As discussed earlier, the first eigenvalue of the Kennaugh matrix can be interpreted as the total available power in the backscattered wave before it reaches the receiver. The maximal received power that we have calculated represents 
the maximal receivable power from the polarized component of the backscattered wave plus the received power from the unpolarized component, which is always half of the available power in the unpolarized part. The minimal received power, of course, is from the unpolarized part of the backscattered wave. The last column of Table III indicates that the sum of the maximal and minimal received powers is nearly equal to the first eigenvalue of the Kennaugh matrix, which supports the above interpretations. Therefore, we can define the following parameter, named as $D p$, to describe a target's ability to generate an unpolarized component in the scattered wave:

$$
D p=\left(\lambda_{1}(\mathbf{K})-P_{\max }\right) / \lambda_{1}(\mathbf{K})
$$

where $\lambda_{1}(\mathbf{K})$ is the first eigenvalue of the Kennaugh matrix, and $P_{\max }$ is the maximal received power. In comparison, we introduce the fractional polarization defined in [13], which is highly correlated with the degree of polarization of the scattered wave, i.e.,

$$
F=\left(P_{\max }-P_{\min }\right) /\left(P_{\max }+P_{\min }\right)
$$

The three parameters $P_{\min }, D p$, and $F$ have similar implications. The minimal received power is the pedestal height of the polarization signature graphics for a target [1], which is due to spatial variations in the observed scattering properties and can be interpreted in terms of the presence of an unpolarized component in the return wave [2]. A small pedestal height (small minimal received power) means that there is a large amount of a polarized component in the scattered wave, which, thus, corresponds to a small $D p$. The large polarized part of the return wave means that variations in the antenna polarization (receive or transmit) will cause relatively large variations in the received power; therefore, the fractional polarization $F$ is large. With the pedestal height increasing, $D p$ becomes larger, and $F$ becomes smaller.

Among the five land-cover types, water has the smallest minimal received power. This may be explained by the Bragg scattering of the sea surface at large incidence angles (the far range where the AOI $\mathrm{H}$ location has an incidence angle of $58^{\circ}$ ), which is characterized by a low polarimetric entropy and, thus, by a low unpolarized backscattered energy [14]. The much larger minimal received powers from forests and potato fields indicate that there exist considerable variations of the scattering properties among the observed elementary scatterers. The volume scattering of the forest canopy and potato stems and leaves may cause a significant portion of unpolarized return, which accounts for the very large $D p$ and very small fractional polarization $F$. Rapeseed and winter wheat show similar features in all parameters. Although the three parameters carry consistent implications, the relative dynamic range of $D p$ is the largest. This suggests that $D p$ is very sensitive to the variations of scattering properties and can better characterize the scattering process of the illuminated target.

\section{CONCLUSION}

This letter has proposed a numerical method to calculate the maximal and minimal received powers for a target from polarimetric SAR data. The method has been validated by an experiment based on an AIRSAR L-band image. In comparison with widely used computation methods, e.g., the Schuler method based on the eigenvalues of the Graves matrix, the proposed cross-step iteration method is more computationally efficient. More importantly, the cross-step iteration method can derive both the maximal and minimal received powers directly, whereas many other analytic-solution-based methods, including the Schuler method, can only calculate the maximal received power.

This letter has analyzed the relationship between the first eigenvalue of the Kennaugh matrix and optimal received powers. A parameter defined as the relative difference between the first eigenvalue and the maximal received power has been proposed to account for the ability of a target to generate an unpolarized return wave. The parameter has been found to be very sensitive to variations of scattering properties and, therefore, could be useful for terrain-cover-type discrimination. Further studies are needed to quantitatively evaluate the potential and limits of the maximal and minimal received powers and the derived parameters under different radar frequencies for target recognition and terrain discrimination.

\section{REFERENCES}

[1] W.-M. Boerner, C.-L. Liu, and X. Zhang, "Comparison of the optimization procedures for the $2 \times 2$ Sinclair and the $4 \times 4$ Mueller matrices in coherent polarimetry and its application to radar target versus background clutter discrimination," Int. J. Adv. Remote Sens., vol. 2, no. 1, pp. 55-82, Jan. 1993.

[2] H. A. Zebker and J. J. Van Zyl, "Imaging radar polarimetry: A review," Proc. IEEE, vol. 79, no. 11, pp. 1583-1606, Nov. 1991.

[3] R. Touzi, W. M. Boerner, J. S. Lee, and E. Lueneburg, "A review of polarimetry in the context of synthetic aperture radar: Concepts and information extraction," Can. J. Remote Sens., vol. 30, no. 3, pp. 380-407, Jun. 2004.

[4] R. Touzi, S. Goze, T. Le Toan, A. Lopes, and E. Mougin, "Polarimetric discriminators for SAR images," IEEE Trans. Geosci. Remote Sens. vol. 30, no. 5, pp. 973-980, Sep. 1992.

[5] S. L. Durden, J. J. Van Zyl, and H. A. Zebker, "The unpolarized component in polarimetric radar observations of forested areas," IEEE Trans. Geosci. Remote Sens., vol. 28, no. 2, pp. 268-271, Mar. 1990.

[6] A. B. Kostinski, B. D. James, and W. M. Boerner, "Optimal reception of partially polarized waves," J. Opt. Soc. Amer. A, Opt. Image Sci., vol. 5, no. 1, pp. 58-64, Jan. 1988.

[7] ESA, PolSARPro: The Polarimetric SAR Data Processing and Educational Tool2005. [Online]. Available: http://earth.esa.int/polsarpro/ acknowledgements.html

[8] CCRS, Advanced Radar Polarimetry Tutorial2004. [Online]. Available: http://www.ccrs.nrcan.gc.ca/resource/tutor/polarim/pdf/polarim_e.pdf

[9] E. Luneburg and W. M. Boerner, "Optimal polarizations in radar polarimetry," in Proc. PIERS, ESA-ESTEC, Noordwijk, The Netherlands, 1994, pp. 1813-1816.

[10] R. Horn and C. Johnson, Matrix Analysis. Cambridge, U.K.: Cambridge Univ. Press, 1985, ch. 5.

[11] D. L. Schuler and J. S. Lee, "A microwave technique to improve the measurement of directional ocean wave spectra," Int. J. Remote Sens., vol. 16, no. 2, pp. 199-215, Jan. 1995.

[12] W. M. Boerner, H. Mott, E. Lunenburg, C. Livingstone, B. Brisco, R. J. Brown, and J. S. Patterson, "Polarimetry in remote sensing: Basic and applied concepts," in Manual of Remote Sensing, vol. 2, Principles and Applications of Imaging Radar., 3rd ed. New York: Wiley, 1998, ch. 5, pp. 282-304.

[13] H. A. Zebker, J. J. Van Zyl, and D. N. Held, "Imaging radar polarimetry from wave synthesis," J. Geophys. Res., vol. 92, no. B1, pp. 683-701, Jan. 1987.

[14] J. J. Van Zyl, H. A. Zebker, and C. Elachi, "Imaging radar polarization signatures: Theory and observation," Radio Sci., vol. 22, no. 4, pp. 529543, Jul./Aug. 1987. 